\title{
Anti-BP180 and anti-BP230 enzyme-linked immunosorbent assays for diagnosis and disease activity tracking of bullous pemphigoid: A prospective cohort study
}

\author{
Kumutnart Chanprapaph, ${ }^{1}$ Vipawee Ounsakul, ${ }^{1}$ Duangrat Pruettivorawongse, ${ }^{1}$ Kunlawat Thadanipon ${ }^{1,2}$
}

\begin{abstract}
Background: Autoantibodies against BP180 and BP230 play major roles in bullous pemphigoid (BP). We are the first to describe the values of serum anti-BP180 IgG and anti-BP230 IgG enzyme-linked immunosorbent assays (ELISA) for diagnosis and disease monitoring of $\mathrm{BP}$ among Thai patients.
\end{abstract}

Objectives: We aimed to determine the diagnostic performance of anti-BP180 IgG and anti-BP230 IgG in BP, to correlate disease activity with autoantibody levels through follow-ups, and to relate BP comorbidities with disease activity and autoantibody levels.

Methods: Consecutive patients suspected of having BP were included. Skin biopsy, direct immunofluorescence, and serum anti-BP180 IgG and anti-BP230 IgG tests were performed. BP disease area index (BPDAI) was evaluated at diagnosis and throughout follow-ups.

Results: Of 131 patients, 68 were diagnosed with BP, and 63 were included as controls. Sensitivity and specificity of serum anti-BP180 IgG were $69.1 \%$ and $90.5 \%$, respectively, while those of serum anti-BP230 IgG were $55.9 \%$ and $85.5 \%$, respectively. Using anti-BP180 and anti-BP230 IgG antibodies resulted in a 7\% increase in sensitivity compared with using anti-BP180 IgG antibody alone. Significant correlation with BPDAI was found for both autoantibodies at diagnosis but only for anti-BP180 IgG at follow-ups $(p=0.013)$. BP patients with positivity to anti-BP180 or anti-BP230 IgG had significantly higher BPDAI than did those without $(p=0.005)$. BP was associated with neurological diseases $(p=$ $0.025)$, while patients with diabetes had higher disease activity $(p=0.010)$.

Conclusions: While both serum autoantibodies are useful for diagnosing BP in patients with suspicious clinical features, only anti-BP180 IgG allowed prediction of disease activity over time.

Key words: anti-BP180 ELISA, anti-BP230 ELISA, bullous pemphigoid, BPAG1, BPAG2

\author{
Affiliations: \\ Division of Dermatology, Department of Medicine, \\ Faculty of Medicine Ramathibodi Hospital, Mahidol University, \\ Bangkok, Thailand \\ Section for Clinical Epidemiology and Biostatistics, Faculty of \\ Medicine Ramathibodi Hospital, Mahidol University, Bangkok, \\ Thailand
}

\section{Introduction}

Bullous pemphigoid (BP) is the most common autoimmune bullous disease that affects elderly patients $(>60$ years old). ${ }^{1}$ It is characterized by the presence of tissue-bound and circulating autoantibodies directed against the structural components of the cutaneous basement membrane zone. ${ }^{2}$ These autoantibodies predominantly target two hemidesmosomal proteins, namely, BP180 (BPAG2 or type XVII collagen) and

\section{Corresponding author:}

Kunlawat Thadanipon

Division of Dermatology, Department of Medicine, and Section for Clinical Epidemiology and Biostatistics, Faculty of Medicine

Ramathibodi Hospital, Mahidol University

270 Rama VI Road, Rajthevi, Bangkok, Thailand 10400

E-mail: kunlawat.tha@mahidol.ac.th

BP230 (BPAG1). ${ }^{3}$ BP180 is a transmembrane protein in which the noncollagenous extracellular portion, namely, the NC16A domain, is the main immunogenic target of the autoantibodies. ${ }^{4}$ The role of BP180 antibody in the pathogenesis of BP is well elucidated, and the antibody levels have been reported to fluctuate with disease severity. ${ }^{5-7}$ Recent evidence has also indicated that BP patients negative for antibodies against 
BP180 NC16A domain have milder phenotypes. ${ }^{8}$ BP230 is an intracellular protein that belongs to the plakin family. ${ }^{9}$ Although the role of BP230 antibodies in the pathogenesis of $\mathrm{BP}$ and the correlation between anti-BP230 antibody levels and disease severity are not clearly established, the C-terminal domain of BP230 has been proposed as a major antigenic site recognized by BP sera. ${ }^{10-14}$

Detection of serum autoantibodies can be assessed by standard indirect immunofluorescence (IIF) or salt-split skin technique. However, these methods are time-consuming, difficult to standardize, and highly dependent on the operator's skills. Several enzyme-linked immunosorbent assays (ELISAs) have been established with varying reported sensitivity and specificity, based on the techniques and recombinant proteins used. The ELISAs using bacterial recombinant protein of the BP180-NC16A domain and BP230 C-terminal domain have become commercially available and more convenient to use. Moreover, the level of the anti-BP180 ELISA more accurately parallels the disease activity of BP than does IIF.,15

To our knowledge, there are no available data using antiBP180 IgG and anti-BP230 IgG ELISAs as a diagnostic tool and for disease monitoring of BP in Thai patients. In this study, the main objective is to evaluate the diagnostic performance of anti-BP180 IgG and anti-BP230 IgG ELISAs, and determine their relationship with $\mathrm{BP}$ disease activity at diagnosis and throughout the study. We also compared disease activity scores between BP patients with positive and negative autoantibodies to explore the differences between the 2 groups. Finally, we evaluated the association of BP comorbidities with disease activity and serum levels of anti-BP180 IgG and anti-BP230 IgG.

\section{Methods \\ Study population}

This study was conducted at the Dermatology outpatient department, Ramathibodi Hospital, Mahidol University, Bangkok, Thailand, between November 2014 and December 2016. We included all consecutive cases with clinical suspicion of BP presenting with vesiculobullous, urticarial, erosive, or crusting lesions, as well as elderly patients having urticaria, pruritic, or prurigo-like skin conditions. Skin biopsy for histopathology, direct immunofluorescence (DIF), and anti-BP180 IgG and anti-BP230 IgG serum level measurements were performed in all patients. Data including age at diagnosis, gender, duration of symptoms, and comorbidities were also recorded.

The diagnosis of BP was made in patients possessing all features of the following criteria: subepidermal separation with dermal eosinophilic infiltration from skin histopathology; detection of IgG and/or C3 deposits in a linear pattern along the basement membrane by DIF; and linear IgG on the epidermal side of the skin in salt-split IIF. Patients who did not meet all criteria were included in the non-BP group as controls for the study on diagnostic performance of the ELISA tests.

In the BP group, ELISAs and disease activity scores were assessed throughout the follow-ups when there was clinical improvement, clinical flare, or plan for decrement of immunosuppressant(s).

\section{Anti-BP180 IgG and anti-BP230 IgG enzyme-linked immu- nosorbent assays}

The serum samples were collected from the patients before initiation of systemic immunosuppressive therapy. To assess BP180 IgG and BP230 IgG antibody titers, commercial ELISA kits (anti-BP 180-NC16a-4X and anti-BP230-CF; Euroimmun AG, Lübeck, Germany) were used. The serum samples were diluted to $1: 100$ in accordance with the manufacturer's instructions, and the manufacturer's cutoff value of 20 relative units (RU) per mL was used.

\section{Disease severity assessment}

The severity of $\mathrm{BP}$ was assessed at diagnosis using the $\mathrm{BP}$ Disease Area Index (BPDAI) proposed by the International Pemphigoid Committee in 2012. ${ }^{16}$ The index is composed of both objective measurement of disease severity of skin and mucosa according to anatomical region, with a total score of 120 , and subjective measurement of pruritus severity, with a total score of $30{ }^{16}$

\section{Statistical analysis}

All statistical analyses were performed in Stata version 15.1 (StataCorp. 2017. Stata Statistical Software: Release 15. StataCorp LLC, College Station, TX), except for the mixed-effects quantile regression, which was performed in $\mathrm{R}$ version 3.4.1 (R Core Team. 2017. R: A language and environment for statistical computing. R Foundation for Statistical Computing, Vienna, Austria) using the "lqmm" package. ${ }^{17}$ Categorical variables were described using number and percentage, and tested for association using Pearson's chi-squared or Fisher's exact test. Continuous variables with normal distribution were described using means and standard deviations (SD), and tested for differences using Student t-test, while those with nonnormal distribution were described using medians and interquartile ranges (IQR), and tested for differences using Mann-Whitney U test. Sensitivity, specificity, positive likelihood ratio, and negative likelihood ratio were used to describe the ELISA tests. Receiver operating characteristic (ROC) curve analysis was performed to compare the diagnostic performance of the ELISA tests using the area under the curve (AUC) and to find the optimum cutoff value for diagnosing BP using Youden's index (i.e., sensitivity + specificity - 1). Correlations between anti-BP180 IgG, anti-BP230 IgG, and BPDAI at diagnosis were assessed using Spearman's correlation coefficient $(\rho)$. Mixed-effects quantile regression analysis with adjustment for visit number was performed to determine the associations between autoantibody levels and BPDAI through the follow-ups. A two-sided $p$ value of less than 0.05 was considered statistically significant in all analyses.

\section{Ethics statement}

This study was approved by the ethics board of Faculty of Medicine, Ramathibodi Hospital, Mahidol University (protocol ID 07-58-13), prior to the start of the study and performed according to the Declaration of Helsinki. Written informed consent was obtained from all study participants. 


\section{Results}

\section{Characteristics of patients}

A total of 131 patients were included in this study: 68 BP cases ( 28 men, 40 women; mean \pm SD age: $77.8 \pm 10.4$ years) and 63 non-BP cases (26 men, 37 women; mean \pm SD age: $58.8 \pm 17.4$ years), see Table 1 . There was a slight female predominance in both groups. The mean age of BP patients was significantly older than that of non-BP cases $(p<0.001)$. The median (IQR) durations of symptoms in $\mathrm{BP}$ and nonBP groups were 4 weeks $(0.43,216)$ and 4 weeks $(0.57,24)$, respectively. All BP cases showed characteristic subepidermal separation with dermal eosinophil infiltration and linear deposition of IgG and/or C3 along dermoepidermal junction on DIF, together with salt-split IIF, demonstrating positive IgG in an epidermal pattern. Most BP patients had mild disease, with median (IQR) BPDAI and pruritus scores at diagnosis of $10(1,45)$ and $12(10,20)$, respectively. Almost half of the BP patients (29 patients, $42.6 \%$ ) presented with localized disease. Mucosal involvement, mainly affecting oral mucosa, was observed in 4 of 68 patients (5.9\%).

\section{Table 1. Patients' baseline characteristics}

\begin{tabular}{|c|c|c|c|}
\hline Characteristic & $\begin{array}{c}\text { BP patients } \\
(\mathrm{n}=68) \\
\text { Median (IQR) }\end{array}$ & $\begin{array}{c}\text { Non-BP patients } \\
(\mathrm{n}=63) \\
\text { Median (IQR) }\end{array}$ & $p$ value \\
\hline Age, mean \pm SD & $77.8 \pm 10.4$ & $58.8 \pm 17.4$ & $<0.001$ \\
\hline \multicolumn{4}{|l|}{ Sex, n (\%) } \\
\hline Men & $28(41.2)$ & $26(41.3)$ & 0.991 \\
\hline Women & $40(58.8)$ & $37(58.7)$ & \\
\hline $\begin{array}{l}\text { Duration of } \\
\text { symptoms (weeks) }\end{array}$ & $4(0.4,216)$ & $4(0.4,24)$ & 0.522 \\
\hline BPDAI & $10(1,45)$ & N/A & \\
\hline Pruritus score & $12(10,20)$ & N/A & \\
\hline $\begin{array}{l}\text { Anti-BP180 IgG } \\
(\mathrm{RU} / \mathrm{mL})\end{array}$ & $99.3(2.3,200)$ & $4(2,31.6)$ & $<0.001$ \\
\hline $\begin{array}{l}\text { Anti-BP230 IgG } \\
(\mathrm{RU} / \mathrm{mL})\end{array}$ & $38.9(2,200)$ & $4.5(2,55.1)$ & $<0.001$ \\
\hline
\end{tabular}

BP, bullous pemphigoid; BPDAI, bullous pemphigoid disease area index; N/A, not applicable; RU, relative units

The final diagnoses in the non-BP group were other autoimmune bullous diseases, representing 30.2\% (19/63) of patients. They are composed of pemphigus foliaceus (9), pemphigus vulgaris (7), paraneoplastic pemphigus (1), and bullous systemic lupus erythematosus (1). The remaining $69.8 \%$ (44/63) accounted for other nonimmunobullous diseases, shown in Table 2.

\section{Bullous pemphigoid comorbidities}

Among the comorbidities, only neurological diseases (i.e., cerebrovascular diseases, Parkinson's disease, Alzheimer's disease, dementia, and epilepsy) were significantly associated
Table 2. The final diagnosis of controls

\begin{tabular}{|c|c|}
\hline Diagnosis & n (\%) \\
\hline \multicolumn{2}{|l|}{ Autoimmune diseases } \\
\hline Pemphigus foliaceus & $9(14.3)$ \\
\hline Pemphigus vulgaris & $7(11.1)$ \\
\hline Paraneoplastic pemphigus & $1(1.6)$ \\
\hline Bullous systemic lupus erythematosus & $1(1.6)$ \\
\hline \multicolumn{2}{|l|}{ Other diseases } \\
\hline Pruritic rash (eczema, prurigo nodularis) & $15(23.8)$ \\
\hline Bullous insect bite reaction & $8(12.7)$ \\
\hline Bullous leukocytoclastic vasculitis & $3(4.8)$ \\
\hline Friction blister & $3(4.8)$ \\
\hline Lichen planus variant & $3(4.8)$ \\
\hline Dyshidrosis & $2(3.2)$ \\
\hline Chronic scarring conjunctivitis & $2(3.2)$ \\
\hline Bullous fixed drug eruption & $2(3.2)$ \\
\hline Urticaria & $1(1.6)$ \\
\hline Urticarial vasculitis & $1(1.6)$ \\
\hline Erythema multiforme & $1(1.6)$ \\
\hline Erosive lichen planus & $1(1.6)$ \\
\hline Sweet syndrome & $1(1.6)$ \\
\hline Spongiotic dermatitis & $1(1.6)$ \\
\hline Vasculopathy & $1(1.6)$ \\
\hline
\end{tabular}

with BP compared with controls (odds ratio: 5.19; 95\% CI: $1.10,24.58 ; p=0.025)$. Forty percent of BP patients had at least one neurological disease. Median (IQR) anti-BP180 IgG levels in BP patients with and without neurological diseases were $172.4(11.7,200)$ and $15.3(2,200)$, respectively $(p=$ $0.001)$. When stratified by titer levels, an anti-BP180 IgG antibody titer of $100 \mathrm{RU} / \mathrm{mL}$ or higher was significantly associated with neurological diseases $(p=0.024)$. However, the analysis did not reveal any significant association between anti-BP230 IgG antibody levels and neurological diseases in BP patients.

Regarding disease severity, BP patients who had diabetes mellitus $(n=24)$ had significantly higher disease activity than did nondiabetic patients $(n=44)(p=0.010)$. Median (IQR) BPDAI in BP patients with and without diabetes mellitus was $20(8,29)$ and $7(1,42)$, respectively. Among 24 patients with diabetes mellitus, 5 had administered dipeptidyl peptidase- 4 inhibitors (DPP4i) (2 vildagliptin, 2 linagliptin, and 1 sitagliptin) before the onset of BP. However, there was no statistically significant association between anti-BP180 IgG or anti-BP230 IgG positivity and history of DPP4i use $(p=0.693$ and 0.806 , respectively). 
Table 3. Sensitivity, specificity, positive and negative likelihood ratios, and area under ROC curve of anti-BP180 IgG and anti-BP230 IgG ELISAs

\begin{tabular}{|c|c|c|c|c|c|}
\hline ELISA & $\begin{array}{l}\text { Sensitivity, } \% \\
(95 \% \mathrm{CI})\end{array}$ & $\begin{array}{l}\text { Specificity, }{ }^{a} \% \\
(95 \% \text { CI })\end{array}$ & $\begin{array}{l}\text { Positive likelihood } \\
\text { ratio }^{a}(95 \% \mathrm{CI})\end{array}$ & $\begin{array}{l}\text { Negative likelihood } \\
\text { ratio }^{\mathrm{a}}(\mathbf{9 5 \%} \mathrm{CI})\end{array}$ & $\begin{array}{l}\text { Area under ROC } \\
\text { curve }(95 \% \mathrm{CI})\end{array}$ \\
\hline Anti-BP180 IgG & $69.1(56.7,79.8)$ & $90.5(80.4,96.4)$ & $7.26(3.34,15.80)$ & $0.34(0.24,0.49)$ & $0.892(0.837,0.947)$ \\
\hline Anti-BP230 IgG & $55.9(43.3,67.9)$ & $85.5(74.2,93.1)$ & $3.85(2.03,7.30)$ & $0.52(0.39,0.69)$ & $0.749(0.664,0.834)$ \\
\hline $\begin{array}{l}\text { Anti-BP180 IgG or } \\
\text { Anti-BP230 IgG }\end{array}$ & $76.5(64.6,85.9)$ & $79.0(66.8,88.3)$ & $3.65(2.21,6.02)$ & $0.30(0.19, .047)$ & N/A \\
\hline
\end{tabular}

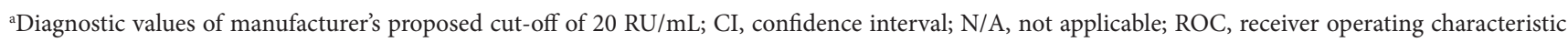

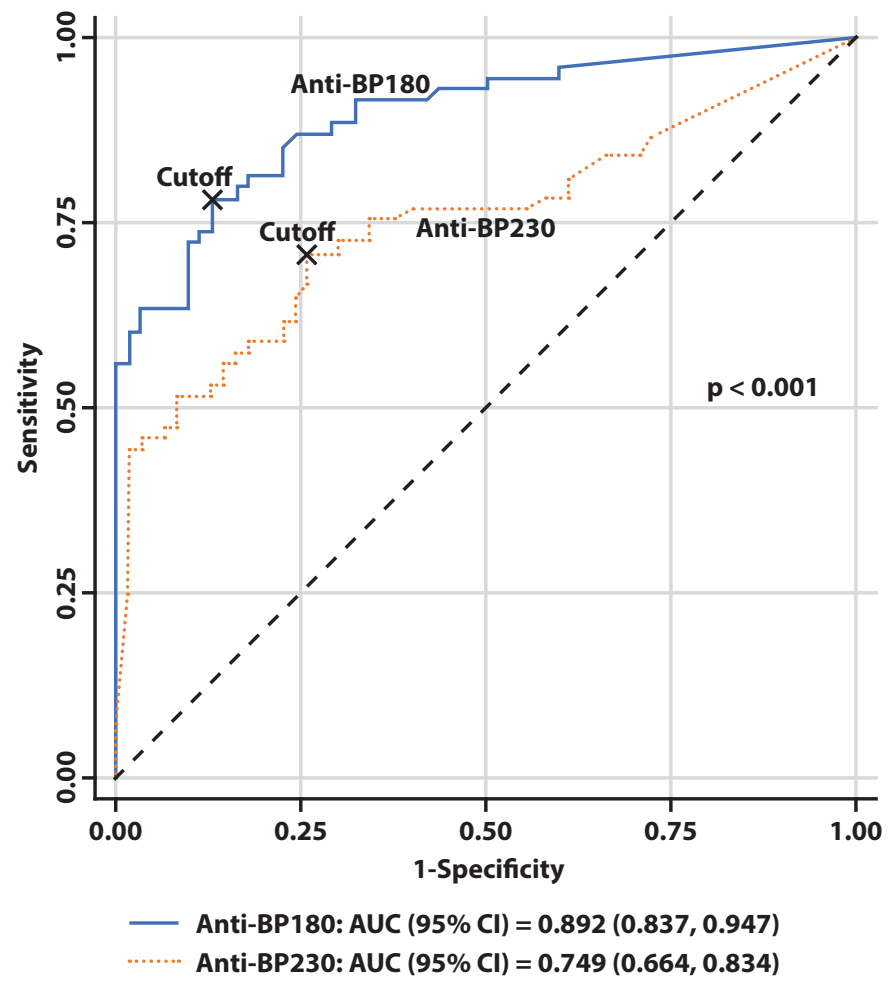

Figure 1. Receiver operating characteristic curves of anti -BP180 IgG and anti-BP230 IgG ELISAs and their optimum cutoffs determined by maximum Youden's index

Table 4. Comparison of $\mathrm{BP}$ disease activity among antiBP180 IgG and/or anti-BP230 IgG positive and negative patients

\begin{tabular}{|lcccc|}
\hline \multicolumn{1}{|c}{ ELISA } & Result & n (\%) & $\begin{array}{c}\text { Median BPDAI } \\
\text { (IQR) }\end{array}$ & $p$ value \\
\hline Anti-BP180 IgG & Positive & $47(69.1)$ & $12(2,44)$ & 0.015 \\
& Negative & $21(30.9)$ & $3(2,8)$ & \\
\hline Anti-BP230 IgG & Positive & $38(55.9)$ & $18(5,44)$ & $<0.001$ \\
\hline $\begin{array}{l}\text { Anti-BP180 IgG } \\
\text { or Anti-BP230 }\end{array}$ & Pegative & $30(44.1)$ & $5(2,16)$ & \\
\hline IgG & Positive & $52(76.5)$ & $12(2,45)$ & 0.005 \\
\hline & Negative & $16(23.5)$ & $2(2,6)$ & \\
\hline
\end{tabular}

BP, bullous pemphigoid; BPDAI, bullous pemphigoid disease area index; IQR, interquartile range

\section{Diagnostic performance of anti-BP180 IgG and anti-BP230 IgG serum levels in $B P$}

Using the $20 \mathrm{RU} / \mathrm{mL}$ cutoff, anti-BP180 IgG antibodies were positive in $47 \mathrm{BP}$ patients and 6 non-BP patients, while anti-BP230 IgG antibodies were positive in $38 \mathrm{BP}$ patients and 9 non-BP patients. The anti-BP180 IgG serum level showed sensitivity of $69.1 \%$ and specificity of $90.5 \%$. The sensitivity and specificity of anti-BP230 IgG were $55.9 \%$ and $85.5 \%$, respectively. In comparison to anti-BP180 IgG alone, the combination of both anti-BP180 IgG and 230 IgG ELISA tests (where positivity to either of the 2 tests was considered a positive result) slightly increased the sensitivity from $69.1 \%$ to $76.5 \%$ (Table 3 ). Of the $68 \mathrm{BP}$ patients, 33 had positive results in both anti-BP180 IgG and anti-BP230 IgG ELISAs, whereas 14 had positive anti-BP180 IgG alone, and 5 had positive anti-BP230 IgG alone. Among the 63 controls, positive anti-BP180 IgG ELISA was found in 4, all of which were diagnosed with pemphigus foliaceus, whereas positive anti-BP230 IgG ELISA was found in the 3 controls with the diagnosis of pemphigus vulgaris.

Serum levels of anti-BP180 IgG and anti-BP230 IgG ELISAs were significantly correlated (Spearman's $\rho=0.633, p<$ 0.001). However, ROC curve analysis revealed that the AUC of anti-BP180 IgG (0.892; 95\% CI: 0.837, 0.947) was significantly higher $(p<0.001)$ than that of anti-BP230 $\operatorname{IgG}(0.749$; 95\% CI: $0.664,0.834$ ), see Figure 1. This signifies that the performance of anti-BP180 IgG was superior to that of anti-BP230 IgG in diagnosing BP. The optimum cutoffs, as determined by the maximum Youden's index (i.e., the cutoffs that yielded the highest sensitivity and specificity simultaneously) for positivity were $\geq 13.5 \mathrm{RU} / \mathrm{mL}$ for anti-BP180 IgG ELISA and $\geq 9.19 \mathrm{RU} / \mathrm{mL}$ for anti-BP230 IgG ELISA. Using these cutoffs would result in sensitivity, specificity, positive likelihood ratio, and negative likelihood ratio of $77.9 \%, 87.3 \%$, 6.138 , and 0.253 , respectively, for anti-BP180 IgG, and 70.6\%, 74.2\%, 2.735, and 0.396, respectively, for anti-BP230 IgG.

\section{The relationship between the serum levels of both ELISAs and $B P$ disease severity at diagnosis}

There was a moderate correlation between serum levels of both autoantibodies and BPDAI at diagnosis, with $\rho$ of 0.563 $(p<0.001)$ for anti-BP180 IgG and $0.488(p<0.001)$ for antiBP230 IgG. 
Table 5. Characteristics and test performance of anti-BP180 and anti-BP230 ELISA assays in the diagnosis of BP.

\begin{tabular}{|c|c|c|c|c|c|c|c|}
\hline \multirow[b]{2}{*}{ Author (year) } & \multirow[b]{2}{*}{ Manufacturer } & \multirow[b]{2}{*}{$\begin{array}{l}\text { Number of } \\
\text { BP patients }\end{array}$} & \multirow[b]{2}{*}{$\begin{array}{l}\text { Number of } \\
\text { controls }\end{array}$} & \multicolumn{2}{|c|}{ Anti-BP180 ELISA } & \multicolumn{2}{|c|}{ Anti-BP230 ELISA } \\
\hline & & & & $\begin{array}{c}\text { Sensitivity } \\
(\%)\end{array}$ & $\begin{array}{l}\text { Specificity } \\
(\%)\end{array}$ & $\begin{array}{c}\text { Sensitivity } \\
(\%)\end{array}$ & $\begin{array}{c}\text { Specificity } \\
\text { (\%) }\end{array}$ \\
\hline 1. Present study & Euroimmun & 68 & 63 & 69.1 & 90.5 & 55.9 & 85.5 \\
\hline 2. Keller $(2016)^{21}$ & MBL & 170 & N/A & 54 & 95 & 48 & 94 \\
\hline 3. Esmaili $(2015)^{19}$ & Euroimmun & 50 & 50 & 88 & 96 & 48 & 96 \\
\hline \multirow[t]{2}{*}{ 4. Damoiseaux $(2012)^{18}$} & Euroimmun & 60 & 57 & 86.7 & 93 & 60 & 82.5 \\
\hline & MBL & 60 & 57 & 80 & 98.2 & 58.3 & 93 \\
\hline 5. Feng $(2008)^{15}$ & MBL & 42 & 42 & 97.6 & 97.6 & N/A & N/A \\
\hline 6. Yoshida $(2006)^{10}$ & MBL & 239 & 430 & 69.9 & 98.8 & 72.4 & 99.5 \\
\hline 7. D’Agosto $(2004)^{20}$ & MBL & 36 & 103 & 66.6 & 100 & N/A & N/A \\
\hline 8. Kobayashi $(2002)^{5}$ & MBL & 84 & 538 & 84.4 & 98.9 & N/A & N/A \\
\hline
\end{tabular}

BP, bullous pemphigoid.

The relationship between the serum levels of both ELISAs and the BPDAI through the follow-up period

Of the $31 \mathrm{BP}$ patients who had immunological follow-up, mean \pm SD follow-up interval was $5.42 \pm 2.79$ months. Ninety percent showed clinical improvement or were planning immunosuppressive drug decrement, while $10 \%$ had disease flare. To determine the associations between autoantibody levels and disease activity along the follow-ups, mixed-effects quantile regression analysis with adjustment for visit number showed that anti-BP180 IgG was significantly associated with BPDAI with a regression coefficient of 0.039 (95\% CI: 0.008 , $0.070 ; p=0.013$ ), whereas anti-BP230 IgG was not (regression coefficient: 0.013 ; 95\% CI: $-0.011,0.037 ; p=0.282$ ). In other words, each $1 \mathrm{RU} / \mathrm{mL}$ increase in anti-BP180 IgG level significantly correlated to an approximately 0.04 point increase in median BPDAI.

The relationship between disease activity among anti-BP180 IgG and anti-BP230 IgG ELISA positive and negative patients

The BPDAI scores were significantly higher in anti-BP180 IgG and anti-BP230 IgG positive compared with negative patients, $p=0.015$ and $<0.001$, respectively (Table 4). Likewise, patients with positive results to either anti-BP180 IgG or anti-BP230 IgG had significantly higher BPDAI than did those who were negative to both autoantibodies $(p=0.005)$.

\section{Discussion}

This is the first study to evaluate the diagnostic performance of anti-BP180 IgG and anti-BP230 IgG ELISAs in BP among Thai patients. The cross-sectional design we used in the first phase of the study, in which comparison was made among all patients with then-unknown definite diagnosis, has the advantage over the case-control design used in most previous reports that compared already diagnosed BP patients with unrelated controls (healthy subjects or patients with other dermatological conditions $)^{18,19}$ by minimizing the overestimation of the measures for diagnostic values (e.g., sensitivity and specificity) of the ELISA tests, i.e., avoiding the spectrum bias associated with the case-control design in a study on diagnostic test. This strengthens the diagnostic means of anti-BP180 IgG and anti-BP230 IgG in a real-life situation where ELISA is used to differentiate BP from other blistering conditions or BP-mimicking diseases in patients whose diagnosis is still unknown.

In this study, the sensitivities of anti-BP180 IgG and anti-BP230 IgG were $69.1 \%$ and $55.9 \%$, respectively. The specificities of anti-BP180 IgG and anti-BP230 IgG were $90.3 \%$ and $85.5 \%$, respectively. Using both antibodies resulted in only a $7 \%$ increase in sensitivity compared with using anti-BP180 IgG alone. This is in line with previous reports. ${ }^{11}$ Prior studies have reported the diagnostic performance of commercially available ELISA tests for the detection of anti-BP180 IgG and anti-BP230 IgG antibodies in BP patients, revealing sensitivities of $54-97.6 \%$, and $48-60 \%$, respectively, and specificities of $94.8-100 \%$ and $96-99 \%$, respectively, as shown in Table 5. Although our results follow those of prior reports, the sensitivity and specificity of both ELISAs were slightly lower when compared to those performed with the same commercial ELISA. ${ }^{18,19}$ We believe that the slightly lower specificity results from the cross-sectional design, as mentioned above.

We also found anti-BP180 IgG and anti-BP230 IgG positivity among patients in the control group, of which approximately one-third were comprised of other autoimmune bullous diseases. Transition from pemphigus to bullous pemphigoid has been reported in the literature. ${ }^{20,21}$ Possible explanations include epitope spreading, antigen cross-reactivity, and chance. This could consequently reduce the specificity of autoantibody testing.

Due to the high specificity of both tests, anti-BP180 IgG and anti-BP230 IgG may be beneficial in establishing the diagnosis of BP in those who may have special precautions for performing skin biopsy, e.g., severe bleeding tendency or allergy to local anesthesia, and in settings where histological or DIF studies are not available. Moreover, according to relatively low diagnostic added values of anti-BP230 IgG (7\% increase 
in sensitivity), serum anti-BP180 IgG ELISA alone could be performed in case of limited financial resources.

Consistent with previous studies, we demonstrated that serum levels of both anti-BP180 IgG and anti-BP230 IgG were significantly correlated with disease severity at diagnosis..$^{11,20,22}$ In terms of the disease activity throughout the course of disease, only anti-BP180 IgG showed significant fluctuation in parallel to the disease activity and reflected the disease severity over time..$^{5,714,15}$ We found that every $1 \mathrm{RU} / \mathrm{mL}$ increase in the serum anti-BP180 IgG level corresponded to an increase in BPDAI score of approximately 0.04. Anti-BP230 IgG showed contrary results, in agreement with most studies that reported no association with disease progression..$^{10,13}$

In this study, we evaluated the diagnostic power by using the manufacturer's suggested cutoff value of $20 \mathrm{RU} / \mathrm{mL}$ for both anti-BP180 IgG and anti-BP230 IgG ELISAs. From the ROC curve analysis using Youden's index, the optimum cutoffs for anti-BP180 IgG and anti-BP230 IgG from our study were $\geq 13.5 \mathrm{RU} / \mathrm{mL}$ and $\geq 9.19 \mathrm{RU} / \mathrm{mL}$, respectively. These cutoffs offer the best balance of sensitivity and specificity, and hence are lower than the manufacturer's suggested values. However, due to our study's small sample size, larger studies with a cross-sectional design are needed to determine more precise and accurate cutoff values.

Our study shows that among patients diagnosed with BP, those with positivity to one or both antibodies had significantly higher disease activity than did those without $(p=$ 0.015 and $p<0.001$ for anti-BP180 IgG and anti-BP230 IgG only, respectively, and $p=0.005$ for either anti-BP180 IgG or anti-BP230 IgG). This supports the notion that autoantibodies attacking BP180 on the noncollagenous NC16A and BP230 $\mathrm{C}$-terminal domain are of pathological relevance and determine severe clinical phenotype. This finding is in line with a previous report where patients with $\mathrm{BP}$ who were negative for BP180 NC16A ELISA had milder clinical profiles. ${ }^{8}$

In terms of BP-associated condition, as expected, we found that neurological diseases were significantly higher in BP patients compared to controls, with an odds ratio of 5.19. This is consistent with the results published to date. ${ }^{23-26}$ Degenerative and cerebrovascular diseases may lead to neural tissue damage and subsequently cause the release of cellular proteins such as BP180 and BP230. This could result in autoimmune trigger, with subsequent immune response that may manifest as BP. Previous studies have suggested the pathogenic role of anti-BP230 and the development of BP. Expression of BP230 has been demonstrated in the human brain, and neural anti-BP230 is associated with neurodegenerative disorders. ${ }^{27,28}$ Taghipour et al. demonstrated that BP patients with neurological disease had autoantibodies to both BP180 and BP230 proteins. ${ }^{29}$ Our study, similar to that of Gambicher et al., revealed that only anti-BP180 was significantly associated with neurological disease. ${ }^{30}$ However, recent evidence failed to demonstrate specific bindings of anti-BP180 antibodies in neural tissue in two different mammalian models. ${ }^{30}$ Therefore, more experimental research is required to elucidate the relationship between BP autoantibodies and the development of neurological diseases.
Regarding other comorbidities, diabetes mellitus has been found to be increasingly associated with BP in the last decade. ${ }^{31} \mathrm{~A}$ recent study showed that DPP4i, especially vildagliptin, is associated with increased risk of $\mathrm{BP} .^{32}$ However, there was no significant association between diabetes mellitus and BP in our patients. Previous reports have found that DPP4i administered preceding the onset of BP targeted the non-NC16A domain in virtually all patients. ${ }^{33,34}$ In contrast, we failed to find an association between negative or positive BP180 IgG ELISA testing on NC16A domain in patients commencing DPP4i. This discrepancy remains to be elucidated and requires accumulation of more cases and further investigations. Interestingly, we demonstrated that BP with diabetes mellitus had significantly higher disease severity in general. A possible explanation to the higher disease severity among BP with diabetes mellitus is the contraindication of using systemic corticosteroid in poorly controlled diabetes mellitus, causing difficulty in the management of moderate to severe BP. Moreover, high incidence $(30 \%)$ of cutaneous infection occurred in BP with diabetes mellitus treated with topical corticosteroids, thereby restricting its extensive use. ${ }^{35}$

The limitations of this study are that the sample size was relatively small and that all subjects were from a single institution.

In conclusion, serum anti-BP180 IgG and anti-BP230 IgG ELISAs can be considered useful and convenient methods for diagnosing BP in patients clinically suspected of having BP. However, serum anti-BP230 IgG offers low added diagnostic value and is not useful for monitoring disease severity; therefore, serum level of anti-BP180 IgG alone could be measured in cases of limited resources. BP patients with positivity to either anti-BP180 IgG or anti-BP230 IgG ELISA had higher disease activity than did those without, and this finding supports the fact that these antibodies determine severe clinical phenotype. BP is associated with neurological conditions, while BP patients with diabetes mellitus had significantly higher disease severity. The significance of DDP4i in association with BP remains to be elucidated.

\section{Conflicts of Interest}

The authors have no conflicts of interest to declare.

\section{Funding}

The authors received no funding for this study.

\section{References}

1. Brick KE, Weaver CH, Lohse CM, Pittelkow MR, Lehman JS, Camilleri MJ, et al. Incidence of bullous pemphigoid and mortality of patients with bullous pemphigoid in Olmsted County, Minnesota, 1960 through 2009. J Am Acad Dermatol. 2014;71(1):92-9.

2. Sardy M, Kostaki D, Varga R, Peris K, Ruzicka T. Comparative study of direct and indirect immunofluorescence and of bullous pemphigoid 180 and 230 enzyme-linked immunosorbent assays for diagnosis of bullous pemphigoid. J Am Acad Dermatol. 2013;69(5):748-53.

3. Stanley JR. Cell adhesion molecules as targets of autoantibodies in pemphigus and pemphigoid, bullous diseases due to defective epidermal cell adhesion. Adv Immunol. 1993;53:291-325.

4. Matsumura K, Amagai M, Nishikawa T, Hashimoto T. The majority of bullous pemphigoid and herpes gestationis serum samples react with the NC16a domain of the $180-\mathrm{kDa}$ bullous pemphigoid antigen. Arch Dermatol Res. 1996;288(9):507-9. 
5. Kobayashi M, Amagai M, Kuroda-Kinoshita K, Hashimoto T, Shirakata Y, Hashimoto K, et al. BP180 ELISA using bacterial recombinant NC16a protein as a diagnostic and monitoring tool for bullous pemphigoid. J Dermatol Sci. 2002;30(3):224-32.

6. Schmidt E, Obe K, Brocker EB, Zillikens D. Serum levels of autoantibodies to BP180 correlate with disease activity in patients with bullous pemphigoid. Arch Dermatol. 2000;136(2):174-8.

7. Tsuji-Abe Y, Akiyama M, Yamanaka Y, Kikuchi T, Sato-Matsumura KC, Shimizu H. Correlation of clinical severity and ELISA indices for the NC16A domain of BP180 measured using BP180 ELISA kit in bullous pemphigoid. J Dermatol Sci. 2005;37(3):145-9.

8. Nakama K, Koga H, Ishii N, Ohata C, Hashimoto T, Nakama T. Clinical and Immunological Profiles of 14 Patients With Bullous Pemphigoid Without IgG Autoantibodies to the BP180 NC16A Domain. JAMA Dermatol. 2018;154(3):347-50.

9. Hamada T, Nagata Y, Tomita M, Salmhofer W, Hashimoto T. Bullous pemphigoid sera react specifically with various domains of BP230, most frequently with C-terminal domain, by immunoblot analyses using bacterial recombinant proteins covering the entire molecule. Exp Dermatol. 2001;10(4):256-63.

10. Yoshida M, Hamada T, Amagai M, Hashimoto K, Uehara R, Yamaguchi $\mathrm{K}$, et al. Enzyme-linked immunosorbent assay using bacterial recombinant proteins of human BP230 as a diagnostic tool for bullous pemphigoid. J Dermatol Sci. 2006;41(1):21-30.

11. Charneux J, Lorin J, Vitry F, Antonicelli F, Reguiai Z, Barbe C, et al. Usefulness of BP230 and BP180-NC16a enzyme-linked immunosorbent assays in the initial diagnosis of bullous pemphigoid: a retrospective study of 138 patients. Arch Dermatol. 2011;147(3):286-91.

12. Blocker IM, Dahnrich C, Probst C, Komorowski L, Saschenbrecker S, Schlumberger W, et al. Epitope mapping of BP230 leading to a novel enzyme-linked immunosorbent assay for autoantibodies in bullous pemphigoid. Br J Dermatol. 2012;166(5):964-70.

13. Le Sache-de Peufeilhoux L, Ingen-Housz-Oro S, Hue S, Sbidian E, Valeyrie-Allanore L, Ortonne N, et al. The value of BP230 enzyme-linked immunosorbent assay in the diagnosis and immunological follow-up of bullous pemphigoid. Dermatology. 2012;224(2):154-9.

14. Lee EH, Kim YH, Kim S, Kim SE, Kim SC. Usefulness of Enzyme-linked Immunosorbent Assay Using Recombinant BP180 and BP230 for Serodiagnosis and Monitoring Disease Activity of Bullous Pemphigoid. Ann Dermatol. 2012;24(1):45-55.

15. Feng S, Wu Q, Jin P, Lin L, Zhou W, Sang H, et al. Serum levels of autoantibodies to BP180 correlate with disease activity in patients with bullous pemphigoid. Int J Dermatol. 2008;47(3):225-8.

16. Murrell DF, Daniel BS, Joly P, Borradori L, Amagai M, Hashimoto $\mathrm{T}$, et al. Definitions and outcome measures for bullous pemphigoid: recommendations by an international panel of experts. J Am Acad Dermatol. 2012;66(3):479-85.

17. Geraci M. Linear Quantile Mixed Models: The $\{\mathrm{lqmm}\}$ Package for Laplace Quantile Regression. J Stat Softw. 2014;57(13):1-29.

18. Damoiseaux J, van Rijsingen M, Warnemunde N, Dahnrich C, Fechner K, Tervaert JW. Autoantibody detection in bullous pemphigoid: clinical evaluation of the EUROPLUS Dermatology Mosaic. J Immunol Methods. 2012;382(1-2):76-80.

19. Esmaili N, Mortazavi H, Kamyab-Hesari K, Aghazadeh N, Daneshpazhooh M, Khani S, et al. Diagnostic accuracy of BP180 NC16a and BP230-C3 ELISA in serum and saliva of patients with bullous pemphigoid. Clin Exp Dermatol. 2015;40(3):324-30.
20. Thoma-Uszynski S, Uter W, Schwietzke S, Hofmann SC, Hunziker T, Bernard P, et al. BP230- and BP180-specific auto-antibodies in bullous pemphigoid. J Invest Dermatol. 2004;122(6):1413-22.

21. Recke A, Rose C, Schmidt E, Brocker EB, Zillikens D, Sitaru C. Transition from pemphigus foliaceus to bullous pemphigoid: intermolecular B-cell epitope spreading without IgG subclass shifting. J Am Acad Dermatol. 2009;61(2):333-6.

22. Roussel A, Benichou J, Randriamanantany ZA, Gilbert D, Drenovska K, Houivet E, et al. Enzyme-linked immunosorbent assay for the combination of bullous pemphigoid antigens 1 and 2 in the diagnosis of bullous pemphigoid. Arch Dermatol. 2011;147(3):293-8.

23. Kulthanan K, Chularojanamontri L, Tuchinda P, Sirikudta W, Pinkaew S Prevalence and clinical features of Thai patients with bullous pemphigoid. Asian Pac J Allergy Immunol. 2011;29(1):66-72.

24. Casas-de-la-Asuncion E, Ruano-Ruiz J, Rodriguez-Martin AM, Velez Garcia-Nieto A, Moreno-Gimenez JC. Association between bullous pemphigoid and neurologic diseases: a case-control study. Actas Dermosifiliogr. 2014;105(9):860-5.

25. Lai YC, Yew YW, Lambert WC. Bullous pemphigoid and its association with neurological diseases: a systematic review and meta-analysis. J Eur Acad Dermatol Venereol. 2016;30(12):2007-15.

26. Yu Phuan CZ, Yew YW, Tey HL. Bullous pemphigoid and antecedent neurological diseases: An association with dementia. Indian J Dermatol Venereol Leprol. 2017;83(4):457-61.

27. Seppanen A ST, Hofmann SC, Majamaa K, Alafuzoff I. Distribution of collagen XVII in the human brain. Brain Res. 2007:50-6.

28. Feldrihan V LE, Florea F, Cristea V, Popescu O, Sitaru C, Chiriac MT. IgG antibodies against immunodominant C-terminal epitopes of BP230 do not include skin blistering in mice. Hum Immunol. 2014;75:354-63.

29. Taghipour K CC, Bhogal B, Groves RW, Venning V, Wojnarowska F Immunopathological characteristics of patients with bullous pemphigoid and neurological disease. J Eur Acad Dermatol Venereol. 2014;28(5) 567-73.

30. Gambicher T SH, Hoxtermann S, Schmitz L, Altmeyer P, Teegen B. Neurological disorders in patients with bullous pemphigoid: clinical and experimental investigations. J Eur Acad Dermatol Venereol. 2015;29(9): 1758-62.

31. Fania L, Di Zenzo G, Didona B, Pilla MA, Sobrino L, Panebianco A, et al. Increased prevalence of diabetes mellitus in bullous pemphigoid patients during the last decade. J Eur Acad Dermatol Venereol. 2018;32(4):e153-e4.

32. Benzaquen M, Borradori L, Berbis P, Cazzaniga S, Valero R, Richard MA, et al. Dipeptidyl peptidase IV inhibitors, a risk factor for bullous pemphigoid Retrospective multicenter case-control study from France and Switzerland. J Am Acad Dermatol. 2018;78(6):1090-6.

33. Izumi K, Nishie W, Mai Y, Wada $M$, Natsuga $K$, Ujiie $H$, et al Autoantibody Profile Differentiates between Inflammatory and Noninflammatory Bullous Pemphigoid. J Invest Dermatol. 2016;136(11): 2201-10.

34. Mai Y, Nishie W, Izumi K, Yoshimoto N, Morita Y, Watanabe M, et al. Detection of anti-BP180 NC16A autoantibodies after the onset of dipeptidyl peptidase-IV inhibitor-associated bullous pemphigoid: a report of three patients. Br J Dermatol. 2018;179(3):790-1.

35. Boughrara Z, Ingen-Housz-Oro S, Legrand P, Duong TA, Roujeau IC [Cutaneous infections in bullous pemphigoid patients treated with topical corticosteroids]. Ann Dermatol Venereol. 2010;137(5):345-51. French. 